staff and patients, breaking down initial hostility and lack of understanding and the division between "them" and "us." There was a remarkable absence of disturbances and of demand for sedatives. Of interest was the request by discharged prisoners to be allowed to visit the unit after discharge and the marked change in attitude of the disciplinary officers.

Clearly such an inpatient unit, whether in prison or in hospital, can be no more than an initiation programme; its success must stand or fall with a planned comprehensive aftercare and community service. The planning of such an aftercare programme, involving also the unit staff, was in progress when unfortunately, owing to administrative problems-namely, the current shortage of prison officers-the therapeutic programme had to be curtailed, one hopes only temporarily. In the view of staff and patients, who all bitterly regretted the interruption of the work, the "experiment" has undoubtedly been successful, largely owing to the enthusiasm and dedication of the prison officers. It has indicated the feasibility of setting up a therapeutic community with a therapeutic atmosphere even within the confines of a prison. For those addicts who land in prison (and one may hope that their numbers will gradually decrease) the therapeutic community approach certainly seems to be much more promising than other approaches in current use, and in my own view deserves further trials in other selected prisons under the supervision of interested staff.

The above is the personal view of the writer only, and is not necessarily the view of the Home Office or its Prison Department.

-I am, etc.,

St. Bernard's Hospital

M. M. GLatt

Southall, Middlesex

1 Glatt, M. M., British fournal of Addiction, 1969 64, 165.

Glatt, M. M., The Alcoholic and the Help he

\section{Tenckhoff Catheter for Long-term Peritoneal Dialysis}

SIR,-In reference to the paper by Dr. P. G. Lankisch and others (22 December, p. 712) we would like to make the following comments.

To date we have employed the indwelling Tenckhoff catheter in 13 patients treated by long-term peritoneal dialysis. Dialysis was initially carried out with manual exchange of 2-litre bottles of dialysate, similar to the procedure reported by Dr. Lankisch and his colleagues. Subsequently we have used a closed automated dialysate delivery system. Episodes of peritonitis occurred 13 times in 27 patient-months using the manual system and 11 times in 50 patient-months with the closed system. Five of the latter episodes oocurred in one patient who totally ignored sterile technique; many other instances of peritonitis were due to obvious breaks in sterile procedure. These observations agree with those of Tenckhoff and Curtis.1 Thus peritonitis can be avoided or greatly minimized by the use of a closed autamated system for dialysate delivery and riqid adherence to sterile techniques. Moreover, we found it necessary to remove or replace a catheter only once because of malfunction related to infection. When recognized early, episodes of peritonitis were readily managed by continuous peritoneal dialysis for 4-10 days using fluid containing the appropriate antibiotic.

These observations indicate that longterm peritoneal dialysis has a place in the management of terminal renal failure. It is especially indicated in patients with medical contraindications to haemodialysis, those lacking vascular access, and as an alternative to haemodialysis in hospital for patients who cannot be trained for haemodialysis at home. In the last group home peritoneal dialysis may provide better rehabilitation as it can easily be performed at night during sleep; also, it is less expensive than haemodialysis carried out in a centre.-We are, etc.,

Michael BlumenKRANTZ DESMOND J. SHAPIRO JOEL D. KOPPLE JACK W. COBURN

Veterans Administration, Wadsworth Hospital Center

1 Tenckhoff, H., and Curtis, F. K., Transactions of the American Socieis

\section{Tenckhoff Catheter for Long-term} Peritoneal Dialysis

SIR,-Our experience with the use of the permanent Tenckhoff catheter in chronic peritoneal dialysis is significantly different from that of Dr. P. G. Lankisch and others (22 December, p. 712)

During the past three years we have used 78 catheters in 66 patients. In 16 cases the catheter was obstructed after periods varying from 0.5 to 14 months (average 6 months). In

24 patients the catheter is still functioning after periods of 2.5-36.5 months (average 11.2 months). In the remainder the catheter was still functioning after an average period of seven months (0.5-27 months) when peritoneal dialysis was discontinued. Nine patients have been carrying out their peritoneal dialysis at home, using an automatic cycler, for periods of 2-15 months.

During the three-year period a total of 5,067 dialyses were performed. Ascitic fluid, if present, or a sample of the first effluent was sent for culture on each dialysis. Positive cultures were obtained in 40 cases $(0.8 \%)$ and clinical peritonitis developed in $14(0.25 \%)$. Whenever the catheter is obstructed because of peritonitis we do not change the patient to haemodialysis, but instead we continue dialysis, using the temporary catheter and the Dean prosthesis, until all signs of infection clear and then we replace the catheter. ${ }^{1}$ Fourteen patients received a kidney transplant and the catheter was used in the post-transplant period, if it was necessary, without any complication.

In contrast to the conclusions of $\mathrm{Dr}$. Lankisch and his colleagues, we think that the permanent Tenckhoff catheter is ideal for chronic peritoneal dialysis. It can be used for long periods in patients awaiting renal transplantation. We do not use it in patients with borderline renal failure or patients on haemodialysis with complications who require short-term peritoneal dialysis. In these two groups we use the temporary catheter and the Dean prosthesis as we have described elsewhere. 1 Finally, we have noticed that many catheters function satisfactorily even if there is radiological evidence of misplacement.

It is difficult to understand why our results are so different from those of Dr. Lankisch and his colleagues. We are using only 1,000 units of heparin/2 1. dialysate and actually in those patients on home peritoneal dialysis we do not use heparin at all. We do not leave a residual fluid at the end of dialysis, but we fill the catheter with $3 \mathrm{ml}$ of a solution containing 3,000 units of heparin. We agree that strict aseptic technique is the most important factor for successful chronic peritoneal dialysis and it seems that we are more sucoessful in achieving it, as is shown by our lower incidence of positive cultures and clinical peritonitis. The latter may be responsible for our better catheter survival rates.-We are, etc.

D. G. OREOPOULOS H. DEVINE S. IZATT

Metabolic-Renal Unit,

Toronto Western Hospital

1 Bigelow, P., Oreopoulos, D. G., and DeVeber, 1973, io9, 999.

P. BIGELOW

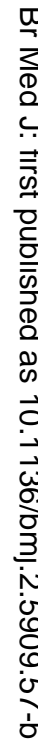

\section{Battering: Dangers of a Backlash}

SIR,-I share the concern of Dr. J. W. Woodward (9 March, p. 452) about the possible harmful effects of precipitant action in the investigation of suspected cases of baby battering. The following case report illustrates the need for restraint and for close liaison between all interested parties.

A 2-year-old girl was brought to the attention of the general practitioner by her parents with an extensive raw and peeling skin lesion approximately $8 \times 15 \mathrm{~cm}$ on the right buttock and upper thigh. The lesion had appeared over a period of 24 hours. The parents' only explanation was that they hours. The parents' only explanation was that they doctor and his partner, both experienced doctors, doctor and his partner, both experienced doctors, conferred and agreed that the lesion was a superficial scald and required treatment in hospital. In "injury" the doctor reluctantly felt obliged to "injury" the doctor reluctantly felt obliged to raise the suspicion of possible child abuse. His
reluctance stemmed from his unique knowledge of reluctance stemmed from his unique knowledge of capable of maliciously inflicting trauma on their child. His dilemma was expressed in confidence to the hospital paediatrician. In the meantime the child had been admitted to the local burns unit where the lesion was treated as a scald

In view of the G.P.'s reservations, initial communication between the paediatrician and the leader of the N.S.P.C.C.'s Special Unit for Battered Babies led to a decision to withhold any precipitan investigation. Observation of the child in hospital investigation. Observation of th: child in hospital over the next 48 hours justified this reticence as two
similar lesions appeared on her legs, and after consimilar lesions appeared on her legs, and after consultation with a dermatologist the conditicn was
diagnosed as the skin disease toxic epidermal necrolysis. Complete resolution of the lesion ensued and the earlier doubts were fortunately never expressed to the parents.

This case illustrates the need expressed by Dr. Woodward for close liaison between all interested parties. Though local ciroumstances may vary, c-ordination of this liaison will generally be the responsibility of the paediatrician, to whom these children ought to be referred. Social agencies rely on the medical opinion, to which the family doctor can make a valuable contribution. Early confrontation with the parents before a discreet investigation of the background circumstances entails the risk of unjustified and extremely distressing accusations whether expressed or implied. These will 
eventually lead to a backlash which will destroy confidence and the necessary need for vigilance and referral of suspected cases of the syndrome. From its nature, this confidence can be built up only slowly, after years of painstaking work, but will be rapidly lost by a few injustices. - I am, etc.,

JAMES K. SARSFIELD

Department of Paediatrics and Child Health,

University of Leeds

\section{Unusual Complication of Use of Sengstaken-Blakemore Tube}

SIR,-We present, below, a previously undescribed hazard of the use of the Sengstaken-Blakemore tube.

The patient was a 55-year-old male alcoholic who presented with bleeding oesophageal varices. During the course of management a SengstakenBlakemore tube was passed and inflated with a Gastrografin and saline solution. ${ }^{12}$ The tube was checked radiologically.

Despite sedation the patient was restless and Despite sedation the patient was restless and
succeeded in pulling the tube so that the oesophageal balloon was partly out of his mouth. The oesophageal balloon was immediately defiated, but no Gastrografin could be aspirated from the gastric balloon. The tube was passed back to the stomach and restored to the traction position. An $x$-ray (fig. 1) showed the gastric balloon still inflated and in position, but the distance between it and the now malpositioned oesophageal balloon showed that the patient's sudden traction had pulled the that the patient's sudden traction had pulled the gastric balloon a considerable distance down the tube. Gastrografin injected via the gastric balloon's filling lumen did not increase the balloon's size, nor could it be reaspirated, showing that the bal

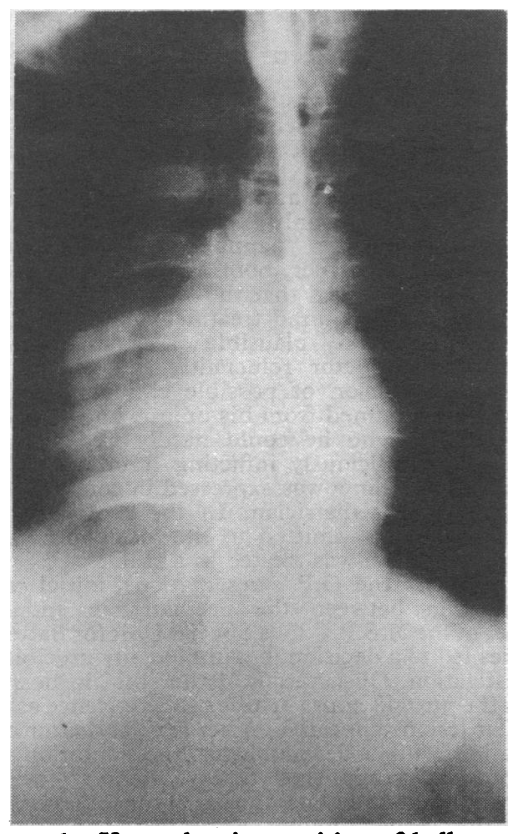

TG. 1-X-ray showing position of balloons after traction by patient.

Later the patient again pulled on the tube and, despite the inflation of the gastric balloon, he succeeded in extracting it, fortunately without damage to the oesophagus. Fig. 2 shows that the gastric to the oesophagus. Fig. 2 shows that the gastric
balloon had now been forced to the tip of the balloon had now been forced to the tip of the is revealed by the two holes of the filling lumen just is revealed by the two holes of the
below the oesophageal balloon.

This case illustrates that the gastric balloon of the Sengstaken-Blakemore tube can be forced away from its filling lumen to a position where it can be neither filled nor deflated. This was done in this case by a restless patient, but there is a danger of the

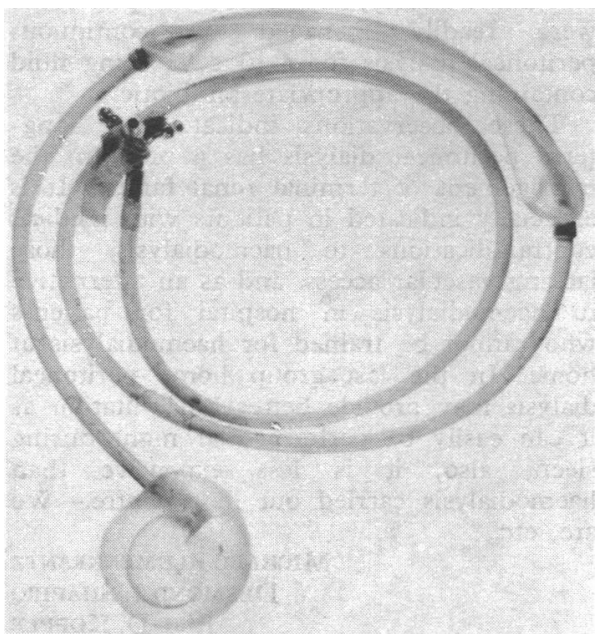

FIG. 2.-Tube after removal by patient.

same complication arising from traction applied therapeutically to the tube-a procedure criticized on other grounds by Pitcher. ${ }^{3}$ This is potentially dangerous for in this situation there may be no way in which the gastric balloon can be safely removed without a laparotomy and gastrotomy.

To some extent the dangers of this complication may be minimized by light sedation, but too heavy sedation increases the risks of inhalation of secretions. The problem could be avoided by redesigning the tube with continuous rubber balloons on the lines of the Foley urinary catheter balloon.-We are, etc.,

C. J. HAWKEY

Northwick Park Hospital

N. R. PEDEN

Harrow, Middlese

1 Read, A. B., Dawson, A. M., Kerr, D. N. S., Turner, M. D., and Sherlock, S., British 2 McPherson, A. I. S., Scottish Medical fournal, 3 Pitcher, J. L., Gastroenterology, 1971, 61, 291.

\section{Apathetic T-3 Toxicosis}

SIR,-Drs. P. D. Fairclough and G. M. Besser (2 March, p. 364) rightly emphasize the occult nature of many cases of hyperthyroidism, be it due to thyroxine or triiodothyronine. Their patient is yet another example of the situation in which a prolonged period of ill health precedes a firm diagnosis of overt hyperthyroidism.

There is evidence that a prethyrotoxic state exists. In elderly subjects, in whom the cardiovascular system is relatively vulnerable, symptoms and signs referable to the heart and circulation may be due to this form of occult hyperthyroidism. The diagnosis can be suspected when only the serum protein-bound iodine is persistently raised and in eight such cases ${ }^{1}$ each developed obvious hyperthyroidism. A similar syndrome may well arise with triiodothyronine, though chemically the diagnosis is even more difficult to establish, as Drs. Fairclough a'nd Besser have shown.-I am, etc.,

\section{London $\mathbf{W} .1$}

CECIL SYMONS 1 Symons, C., Richardson, P. J., and Wood, J. B.,
Patients' Attitudes to Medical Students in General Practice

SIR,-As a student I read with great interest Dr. H. J. Wright's observations (2 March, p. 372) and would like to add some of my own.

I have recently completed an initial course with a group practice in Manchester. Though this was apparently not recognized by its patients as a teaching practice, I very rarely felt that my presence was in any way resented by the patients. On the contrary, many expressed surprise that they might be expected to object (this discoun's the small number of more senior patients who feared that their life-long doctor was being replaced by a young unknown entity and whose fears were rapidly dispelled). Nevertheless, I would have considered the courteous and correct opening to any consultation to be to introduce those present, if necessary explaining the capacity in which they are present, and to ascertain whether the patient objects to their presence. I should have expected such practice to be a common, indeed natural. procedure and was surprised that Dr. Wright thought it necessary to make such a recommendation.

My experience and that of other students, though somewhat limited, indicates that the manner of introduction may have a considerable effect on acceptance by the patient. The term "medical student" implies that the patient is being used as a teaching object, an idea which the presence of two or more students may potentiate, and any such feeling might become a barrier to doctorpatient rapport. The patient-doctor relationship is more personal in general practice than in hospital and therefore to allow the presence of a student at a consultation is an even greater privilege granted by the patient. I believe that the presence of more than one student is an abuse of this privilege.

The introduction of the student as a "student doctor" or, with a little poetic licence, a "young doctor," though meaningless terms, considerably aided acceptance by the patient and also helped to encourage participation by the student. While I was under the guise of a "young doctor frmm the Infirmary" my presence was not objected to during consultation or physical examintiotion, nor indeed was my examination of the patient (which included an internal examination where indicated). The only occasions on which I encountered uneasiness involved patients with psychosocial problems wishing to discuss them with their own doctor; I believe the unease was due to intrusion by another individual rather than specific objection to a student. In such a situation I found it necessary to withdraw and become a very passive observer or to leave the consulting room altogether. -I am, etc.

PHIL G. WIIES

Manchester

\section{Acute Pancreatitis and Diabetic} Ketoacidosis in Hypothermia

SIR,-May we support Dr. R. E. G. Sloan's comments (9 Februarv, p. 245) concerning the need to use arterial blood samples for all biochemical estimations made on hypothermic patients. This has always been our 